\title{
Sublaminar polyester bands for the correction of idiopathic and neuromuscular scoliosis
}

\author{
Federico Canavese, Antoine Samba \\ Pediatric Surgery Department, University Hospital Estaing, Clermont-Ferrand, France \\ Contributions: (I) Conception and design: F Canavese; (II) Administrative support: F Canavese; (III) Provision of study materials or patients: All \\ authors; (IV) Collection and assembly of data: All authors; (V) Data analysis and interpretation: All authors; (VI) Manuscript writing: All authors; (VII) \\ Final approval of manuscript: All authors. \\ Correspondence to: Federico Canavese, MD, PhD. Professor of Pediatric Surgery, Pediatric Surgery Department, University Hospital Estaing, 1 Place \\ Lucie et Raymond Aubrac, 63003 Clermont-Ferrand, France. Email: canavese_federico@yahoo.fr.
}

\begin{abstract}
Recent literature suggests that sublaminar bands (SB) can provide good coronal plane correction, comparable to pedicle screw constructs, as well as good correction in the sagittal plane, even in patients with preoperative hypokyphosis; comparable results have been reported in patients with adolescent idiopathic scoliosis (AIS) and in patients with neuromuscular scoliosis (NMS). Two types of SB constructs can be performed: the band-only construct, indicated for non-ambulatory patients with NMS, and the hybrid construct indicted for ambulatory patients with NMS and for patients with AIS. SB are made of polyester or acrylic material and do provide a safe alternative to sublaminar Luque-type wires (stainless steel) as well as an increased contact area between SB and bone allowing higher corrective forces and reduced laminar fracture risk; the use of SB is not associated with increased risk of neurological injury nor with an increased risk of deep postoperative infection. SB used in a hybrid or in a band-only construct in patients with AIS and NMS, appear to be safe in a trained surgeon hands and can achieve well-balanced spine in both coronal and sagittal planes. This article aimed to provide a review of how SB can restore normal frontal and sagittal spine alignment in patients with AIS and NMS.
\end{abstract}

Keywords: Sublaminar band (SB); hybrid construct; adolescent idiopathic scoliosis (AIS); neuromuscular scoliosis (NMS)

Submitted Aug 23, 2019. Accepted for publication Aug 27, 2019.

doi: 10.21037/atm.2019.08.109

View this article at: http://dx.doi.org/10.21037/atm.2019.08.109

\section{Introduction}

Scoliosis is a three-dimensional deformity of the spine with lateral, antero-posterior and rotational components. The goal of surgery in patients with scoliosis is to restore both frontal and sagittal alignment.

During the past decades, multiple studies have assessed the outcome of different surgical implants such as pedicle screws, hooks, sublaminar wires and hybrid constructs for the management of adolescent idiopathic scoliosis (AIS) and neuromuscular scoliosis (NMS). However, the best type of instrumentation to use in the management of patients with AIS and NMS remains controversial.
Since the introduction of the Harrington's rod, several surgical techniques and instrumentations have been developed to control deformity progression.

In 2009, Mazda et al. (France) have introduced the use of the sublaminar bands (SB) for the treatment of patients with AIS and NMS (1). The principle of reduction with $\mathrm{SB}$ is the so-called posteromedial translation of the spine, initially described by Resina and Alves (Portugal) (2), and later developed and popularized by Luque (Mexico) (3). In Luque's system, two L-shaped rods are placed on either side of the spine and wired to each of the vertebrae. The L-rods are contoured or bent to conform the curve and to provide 
proper sagittal alignment. The wires are threaded through the spinal canal at each vertebral level and are then twisted around the rods on each side of the spine (3).

The hardware to perform spinal correction through SB and segmental fixation has been developed only over the past decade (1) although the principles of posteromedial translation introduced by Resina and Alves (2) and Luque (3) are thirty to forty years older. As a matter of fact, segmental spinal instrumentation with Luque wire fixation has been the standard treatment of NMS for more than three decades (3).

This article aimed to provide a review of how SB can restore normal frontal and sagittal spine alignment in patients with AIS and NMS.

\section{Surgical technique}

The surgical procedure utilizing SB is performed by posterior approach only, with the patient in prone position. Following exposure of the spine, two types of constructs can be performed: the band-only construct and the hybrid construct.

The band-only construct is characterized by two bilateral claws at the upper-instrumented vertebra and SB as thoracic and lumbar anchorages; iliac and/or S1 screws complete the instrumentation. This construct is used in non-ambulatory patients only (NMS) (4). The progressive SB tensioning allows the reduction of the curvature in both frontal and sagittal plane by posteromedial translation (1-3).

The bybrid construct is characterized by two bilateral claws at the upper-instrumented vertebra, several pedicle screws as distal anchorages and SB between upper claws and distal screws. Pedicle screws are free hand inserted and are placed before SB insertion. This construct can used in ambulatory patients with AIS or NMS, and in non-ambulatory patients with NMS (4). The progressive SB tensioning allows the reduction of the curvature in both frontal and sagittal plane by posteromedial translation (1-3).

Segmental SB fixation can be achieved with the same technique in both band-only and hybrid constructs. The polyester SB is passed under the lamina with an insertion technique similar to that of Luque wires, but, with use of the soft polyester bands instead of rigid metal wires, complications sometimes associated with Luque wires have not been observed $(5,6)$. Prior to SB passage, the ligamentum flavum and the inferior edge of the lamina may be partially resected to facilitate insertion of the SB. The SB should be inserted under the lamina, near the midline under the spinous process, in a caudal-to-cephalad direction.
Compared to Luque wires, the band offers a higher surface of bony contact than the wire, thus allowing higher reduction forces (5-7). However, despite the reported safety of the SB, some surgeons remain reluctant at the idea of approaching the canal (7).

Once the SB is passed under the lamina, then all connectors are slid on the rod previously contoured. The set screw locking the connector to the rod stabilizes the construct and avoids undesired rotation during SB tensioning. The reduction is done sequentially by progressively tensioning all the bands with the tension pulley. This gradually translates the spine towards the rods and reduces the deformity by sharing forces between all the implants (posteromedial translation) (8).

Two rods are then connected to the upper claws and to the distal screws in order to obtain a rigid frame. In particular, dual rods ensure both reduction and stabilization of the deformity. Once the desired reduction is achieved, the band is locked within the connector by tightening the band locking set screw. Cobalt-Chrome (CoCr) rods are used more frequently due to the higher rigidity compared to Titanium (Ti) rods. To increase further the stiffness of the construct $6 \mathrm{~mm} \mathrm{CoCr}$ can be used. In patients with NMS with severe pelvic obliquity, iliac and/or S1 screws can be added to both types of constructs and an iliac connector can be used to connect the screws to each rod.

Ponte osteotomies can also be performed at and around the apex of the deformity in all patients in order to soften the spine and to facilitate correction on both sagittal and coronal plane $(4,9)$.

\section{Discussion}

Since their introduction (1), the efficacy of SB for the correction of AIS and NMS has been investigated by several authors. Overall, SB can provide good coronal plane correction, comparable to pedicle screw constructs, as well as good correction in the sagittal plane, even in patients with preoperative hypokyphosis; comparable results have been reported for patients with AIS and NMS (Figures 1-3) (1,4,10-12).

According to some authors, pedicle screw instrumentation offers a greater potential for curve correction in the coronal plane (1,13-15). However, restoring thoracic kyphosis is important to avoid junctional complications at both extremities of the instrumented spine. Posteromedial translation using SB has been shown to more effectively correct hypokyphosis than pedicle screw constructs while 
providing equivalent results in the coronal plane. In addition, the restoration of thoracic kyphosis is generally followed by an adaptation of the adjacent curvatures with improved cervical lordosis and lumbar lordosis (11-15).

Moreover, the relative low density of SB usually required to obtain an efficient and long-lasting correction in patients with scoliosis (AIS and NMS) treated by hybrid constructs should be taken into account in the global cost of scoliosis surgery compared to all pedicle screw-constructs.

\section{SB insertion: is it safe?}

Despite the reported safety of the SB, some surgeons remain reluctant at the idea of approaching the canal (7).

SB are made of polyester or acrylic material and do provide a safe alternative to sublaminar Luque-type wires (made of stainless steel) as well as an increased contact area between SB and bone (SB width: 3 to $5 \mathrm{~mm}$ ) allowing higher corrective forces and reduced laminar fracture risk as metallic wires have a tendency to cut through the bone during correction and allows progressive reduction at multiple apical levels simultaneously (1). In particular, SB have been proven to have the same stress resistance as steel or Ti alloy (metallic) sublaminar wires. Moreover, in contrast to pedicle screws, SB have pullout loads that are independent of bone mineral density. The progressive multiple-level reduction avoids any abrupt traction on the spinal cord and it contributes to reduce the risk of neurological injury further.

Compared to metallic wires, the soft polyester or acrylic SB have been designed to avoid neurological complications during insertion or removal: SB are supple and, once inserted, cannot be inadvertently pushed into the canal. In addition, SB avoid adverse effects related to the presence of metallic wires under the lamina $(14,15)$.

It is important to note that approaching the canal carries the potential risk of neurological injury. To minimize such risk it is possible, as an alternative to sublaminar placement, to wrap the SB around the transverse process of the thoracic vertebrae [sub-transverse bands (STB)]. Hirsch et al. (11) showed that thoracic spine STB and SB can achieve similar correction rate in patients with moderate AIS. Moreover, the STB technique incorporates technical ease with minimal risk of neurological injury and biomechanical stability comparable to SB (1,6,10,13-15). In addition, it is possible that the STB being more lateral than the lamina, might provide greater leverage for correcting the axial deformity (11).
In conclusion, the optimal use of SB requires a strict learning of their insertion technique under the lamina in order to minimize the risk of injuring the cord. SB can be considered as safe as any other spine implant in a trained surgeon's hands.

\section{AIS}

\section{Coronal plane correction}

Posteromedial translation using hybrid constructs associating lumbar pedicle screws, upper thoracic hooks (upper claw) and thoracic SB (apex) provides equivalent coronal plane correction compared to all pedicle screws and hybrid constructs with hooks and pedicle screws (13-17).

A recent literature review by Sales de Gauzy et al. reported that hybrid constructs with SB can achieve a good coronal plane correction of the main thoracic curve $(66 \%$ to $71 \%$ ) with low loss of correction at 24 months follow-up (3\% to $4 \%)(18)$.

La Rosa et al. reported the efficacy and safety of hybrid constructs with SB in correcting coronal deformity and restoring physiological thoracic kyphosis in patients affected by AIS. In particular, they reported $72 \%$ average frontal plane correction and a loss of correction of $7^{\circ} \pm 2^{\circ}$ at 12 months post-surgery (19). The relative flexibility of this type of hybrid construct may explain the loss of correction during the first six months following surgery, while the fusion is not completely acquired. Moreover, most of the time the loss of correction has been shown to be not clinically relevant.

Additionally, Ilharreborde et al. pointed out the reduction in operative time and blood loss in comparison to a group of patients treated with hybrid constructs with hooks in the thoracic region and pedicle screw (5). On the other hand, Palmisani et al. found opposite results and reported the increase in operative time and blood loss in comparison to a group of patients treated with all pedicle crew constructs and concluded that SB can be considered a valid alternative to pedicle screws in presence of hypoplastic pedicles (20).

In our experience, and at least in authors' hands, SB insertion tend to take more time than placing a pedicle screw. A slightly prolonged surgical time should be considered in both patients with AIS and NMS.

\section{Restoration of thoracic kyphosis}

The use of SB has been proven to increase postoperative 
thoracic kyphosis in patients with AIS, particularly in those with hypokyphosis $(1,10,11,16)$. Ilharreborde et al. (5) reviewed 35 patients with AIS treated by hybrid constructs with SB. They were able to restore thoracic kyphosis in $69 \%$ of patients; interestingly, $87.5 \%$ of patients with lordo-scoliosis showed a significant improved of thoracic hypokyphosis to values close to normal $\left(16^{\circ}\right.$ increase of thoracic kyphosis, on average) $(5,18)$.

Similarly, Fletcher et al. have demonstrated that all pedicle screw constructs were less efficient than hybrid constructs with SB in the restoration of thoracic kyphosis in patients with AIS (21). In addition, Hirsch et al. have shown that SB induced a $55 \%$ vertebral derotation (11).

Angelliaume et al. reported that both $\mathrm{CoCr}$ and $\mathrm{Ti}$ rods can achieve similar coronal plane correction in patients treated by posteromedial translation and hybrid constructs. However, $\mathrm{CoCr}$ might be used for better sagittal alignment improvement in hypokyphotic patients (22). Similar results were recently reported by Canavese et al. in patients with NMS (average $24^{\circ}$ increase in thoracic kyphosis and restoration of normal sagittal alignment in $85 \%$ of cases) (4).

Interestingly, Battista et al. reported short term outcomes of hybrid constructs with SB for the treatment of hyperkyphosis $(n=17)$. They found a lower rate of proximal junctional kyphosis (5.8\%) compared to previously published works. They concluded that this technique can protect the fixation within the upper instrumented vertebrae of long segment posterior spine fusions (23).

\section{Sagittal plane changes of adjacent spine segments}

In patients with AIS, the restoration of good sagittal plane alignment is as important as deformity correction on the frontal plane (Figures 1,2) as it directly correlates with the quality of life in adulthood (24).

SB hybrid technique has been shown to provide better sagittal plane correction compared to all-pedicle screw constructs, especially in hypokyphotic patients. Moreover, the restoration of normal thoracic kyphosis results in changes on adjacent sagittal curvatures during follow-up. Blondel et al. reported that lumbar lordosis is a parameter that secondarily adjusts to the improved sagittal balance, induced by the restoration of the thoracic kyphosis. In particular, sagittal alignment changes were seen after 6 months from index surgery (25).

Restoration of normal thoracic kyphosis also induce an augmentation of cervical lordosis, during the whole followup time and starting immediately after surgery. Several authors reported similar results despite the use of different instrumentation and even bracing (26-28).

The restoration of thoracic kyphosis in patients with AIS managed by hybrid construct with SB contributes to improve the sagittal alignment of adjacent spine segments.

\section{NMS}

While the SB technique has already demonstrated benefits in both coronal and sagittal plane correction in patients with AIS, there is a paucity of reports about its use in patients with stiff neuromuscular curves, i.e., NMS.

Albert and LaFleur retrospectively reviewed 29 patients with NMS who underwent segmental spinal instrumentation with SB and pedicle screws (hybrid construct). They reported $69 \%$ average postoperative main curve correction (mean of 28 degrees) and restored sagittal vertical alignment in $97 \%$ of patients, which is comparable to pedicle screw constructs. Moreover, correction was maintained at 29 months follow-up (29). Moreover, Albert and LaFleur pointed out the decrease the potential complications associated with every level transpedicular fixation in patients with a highly dysmorphic and osteoporotic spine (29).

Canavese et al. prospectively assessed 20 patients with NMS and found comparable results. They confirmed that rigid neuromuscular deformities can be successfully managed by posteromedial translation and SB. In particular, Canavese et al. reported $49 \%$ correction in curves with reduction less than $30 \%$ on bending films and found that a physiological kyphosis could be achieved in $88 \%$ of the patients; moreover, a gain of 23 degrees on average on the sagittal plane was recorded in patients with hypokyphosis (4).

Desai et al. retrospectively reviewed a cohort of 21 patients with stiff neuromuscular curves. Desai et al. achieved a mean $39 \%$ correction on the coronal plane which was maintained at last follow-up visit; a mean 2 degrees loss of correction was observed in their group of patients although it was not clinically relevant (30). They also reported two complications associated and five complications non-associated to SB for an overall rate of complications of $33 \%$ (7 out of 21 patients). Complications directly related to the use of sublaminar instrumentation included transient proprioceptive deficit (1 patient), prolonged paraparesis (1 patient), disengagement of the rod from an iliac screw (1 patient), proximal junctional kyphosis (1 patient), non-infected wound drainage (2 patients), and perioperative death (1 patient) (30). Canavese et al. reported a lower rate of complication (20\%) and did not observe 


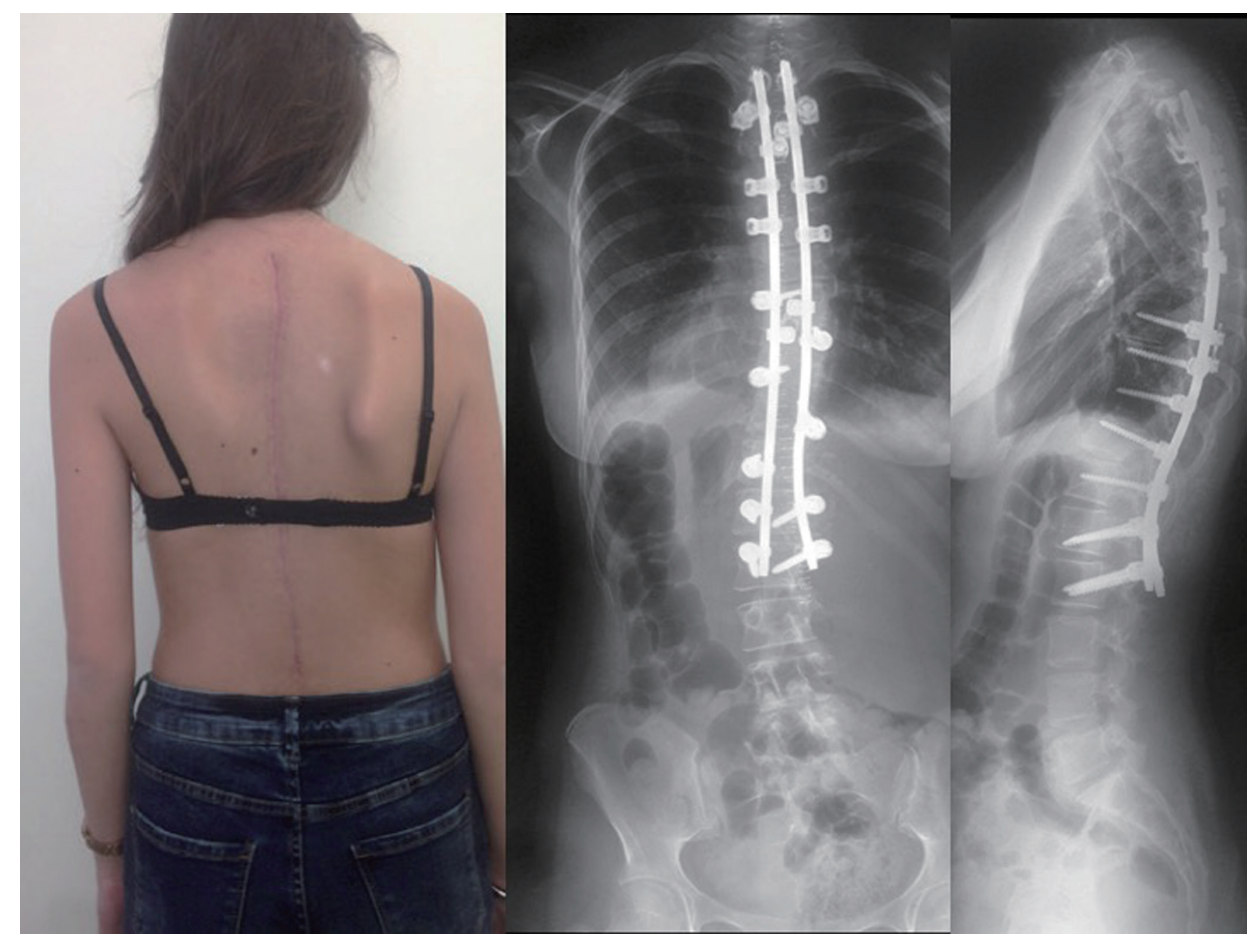

Figure 1 A 15-year-old girl with adolescent idiopathic scoliosis managed by hybrid construct with sublaminar bands in the thoracic spine: good coronal and sagittal plane alignment.

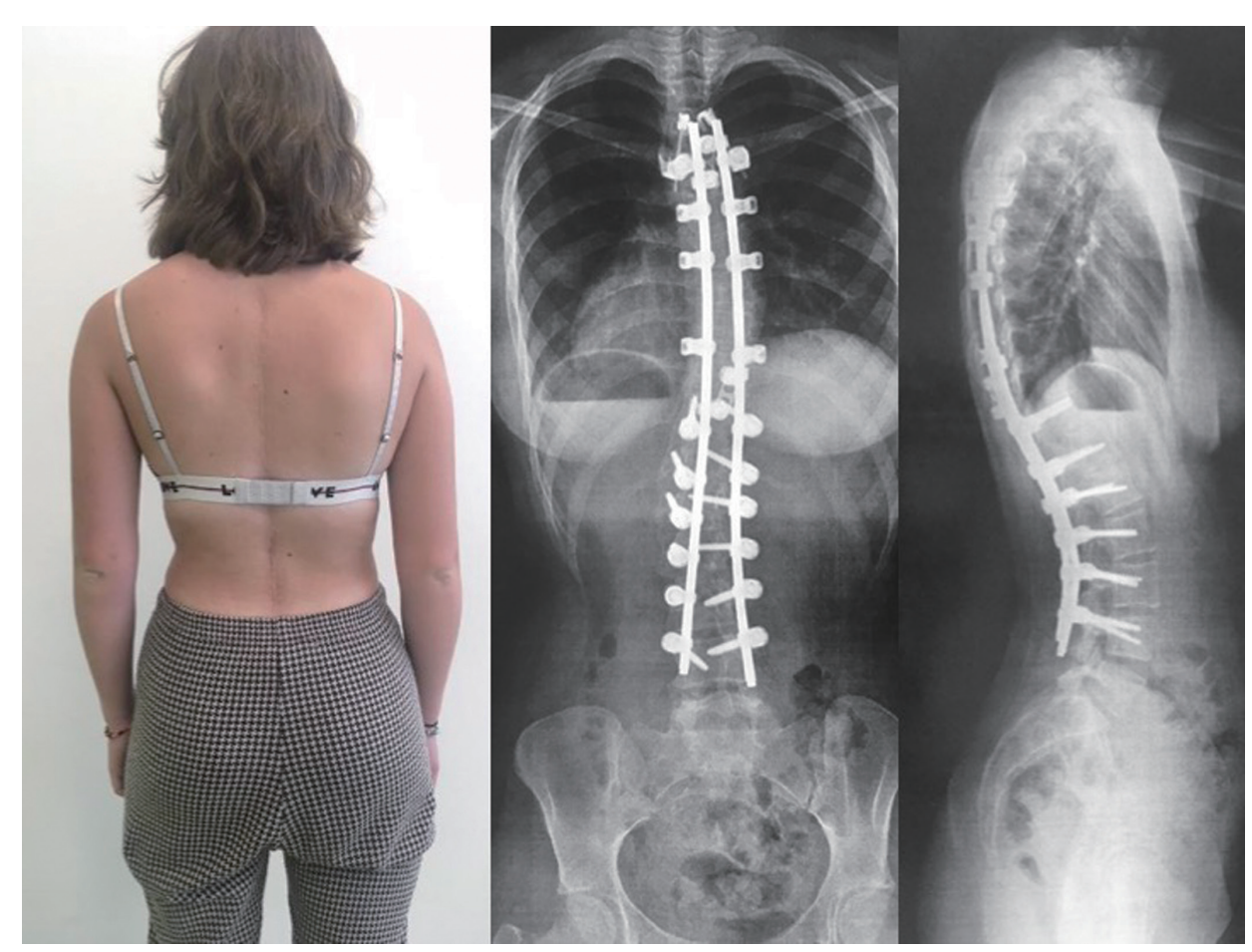

Figure 2 A 16-year-old girl with adolescent idiopathic scoliosis managed by hybrid construct with sublaminar bands in the thoracic spine: good coronal and sagittal plane alignment. 


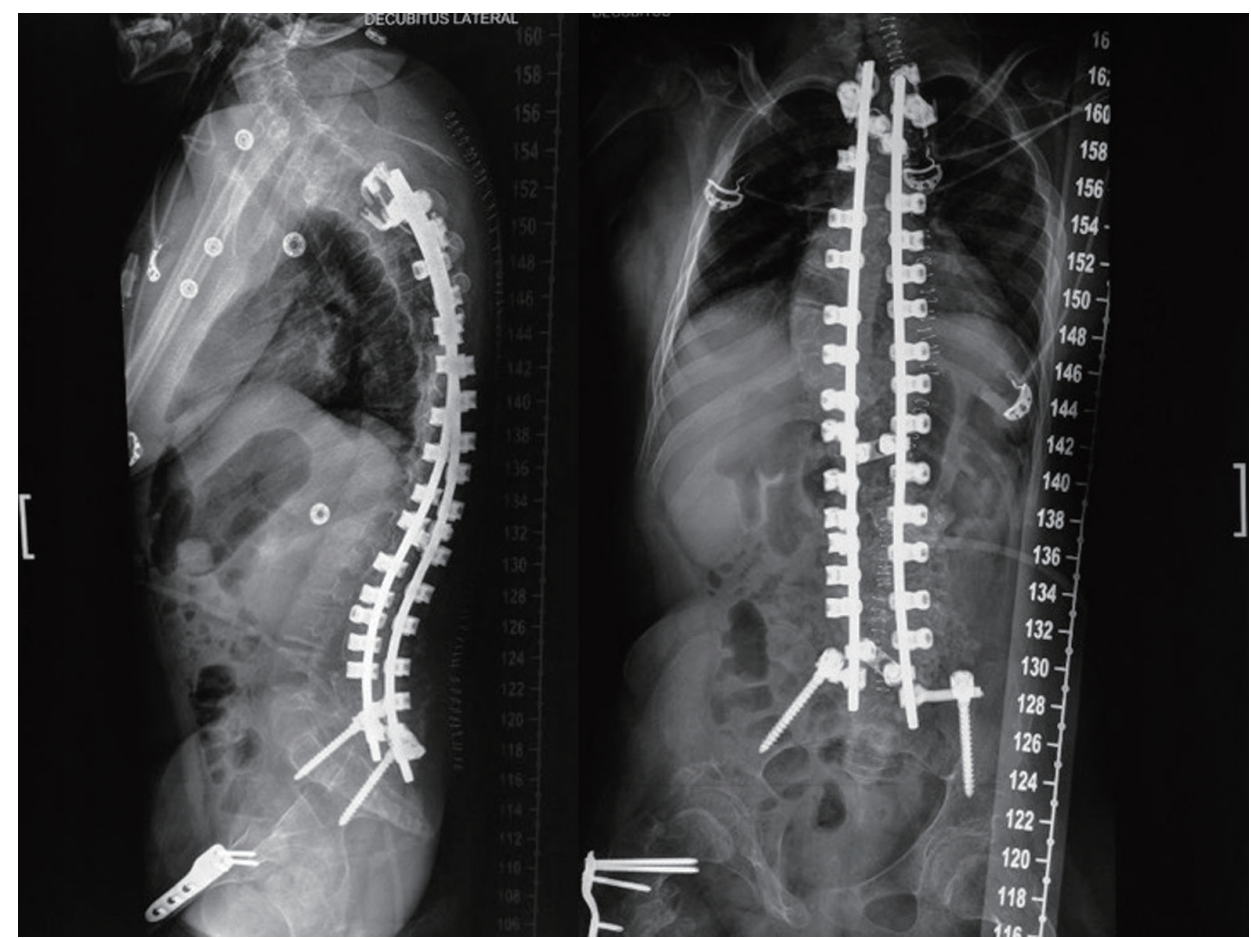

Figure 3 A non-ambulatory 14-year-old girl with neuromuscular scoliosis treated by sublaminar bands-only construct: good coronal and sagittal plane.

any major neurologic complications nor death, during and/or after surgery. Despite these differences, the rate of complications reported by Canavese et al., Desai et al. and Albert and LaFleur is similar to other publications dealing with patients with NMS (4,29-35).

In terms of coronal correction SB achieved a comparable Cobb angle reduction rate than Luque-Galveston instrumentation in neurological scoliosis (50\% in average) and are associated to a lower rate of complications: $20 \%$ instead of $47 \%$ in the Lonstein study (36).

In conclusion, SB used in a hybrid or in a band-only construct (Figure 3) in patients with stiff spinal deformities secondary to neuromuscular conditions, appear to be safe in a trained surgeon hands and can achieve well-balanced spine in both coronal and sagittal planes.

\section{Management of deep post-operative spine infection}

Issa $e t$ al. have shown that the use of SB is not associated with an increased risk of deep post-operative infection nor with a decreased rate of bacterial eradication. They reported
SB removal was needed in 2 out of 594 cases $(0.3 \%)$, and both cases were chronic infections (37).

Canavese et al. found similar results and also highlighted that Vacuum Assisted Closure (VAC) therapy for the management of deep spinal infections can be safely used in patients with AIS and NMS treated by hybrid construct with SB $(38,39)$.

\section{Conclusions}

While achieving correction of deformity in the coronal plane at least equivalent to the best reported results of all-screw or hybrid constructs (hooks), the SB technique provides better correction in the sagittal plane, especially in hypokyphotic patients. SB allow high corrective forces with reduced laminar fracture risk; in addition, the use of SB is not associated with increased risk of neurological injury nor with an increased risk of deep postoperative infection.

\section{Acknowledgments}

None. 


\section{Footnote}

Conflicts of Interest: The authors have no conflicts of interest to declare.

Ethical Statement: The authors are accountable for all aspects of the work in ensuring that questions related to the accuracy or integrity of any part of the work are appropriately investigated and resolved.

\section{References}

1. Mazda K, Ilharreborde B, Even J, et al. Efficacy and safety of posteromedial translation for correction of thoracic curves in adolescent idiopathic scoliosis using a new connection to the spine: the Universal Clamp. Eur Spine J 2009;18:158-69.

2. Resina J, Alves AF. A technique of correction and internal fixation for scoliosis. J Bone Joint Surg Br 1977;59:159-65.

3. Luque ER. Segmental spinal instrumentation for correction of scoliosis. Clin Orthop Relat Res 1982;(163):192-8.

4. Canavese F, Charles YP, Samba A, et al. Safety and efficacy of sublaminar bands and Ponte osteotomies in rigid deformity. Preliminary results in a prospective series of 20 neuromuscular scoliosis patients. J Pediatr Orthop B 2017;26:233-9.

5. Ilharreborde B, Even J, Lefevre Y, et al. Hybrid constructs for tridimensional correction of the thoracic spine in adolescent idiopathic scoliosis: a comparative analysis of universal clamps versus hooks. Spine 2010;35:306-14.

6. Sale de Gauzy J, Jouve JL, Accadbled F, et al. Use of the Universal Clamp in adolescent idiopathic scoliosis for deformity correction and as an adjunct to fusion: 2-year follow-up. J Child Orthop 2011;5:273-82.

7. Wilber RG, Thompson GH, Shaffer JW, et al. Postoperative neurological deficits in segmental spinal instrumentation. A study using spinal cord monitoring. J Bone Joint Surg Am 1984;66:1178-87.

8. Kemal Us A, Yilmaz C, Altay M, et al. Subtransverse process wiring: a new technique of segmental spinal fixation of the thoracic spine or in the treatment of adolescent idiopathic scoliosis. Spine 2001;26:2392-6.

9. Fujita $M$, Diab $M, X u Z$, et al. A biomechanical analysis of sublaminar and subtransverse process fixation using metal wires and polyethylene cables. Spine 2006;31:2202-8.

10. Ferrero E, Pesenti S, Blondel B, et al. Role of thoracoscopy for the sagittal correction of hypokyphotic adolescent idiopathic scoliosis patients. Eur Spine J 2014;23:2635-42.

11. Hirsch C, Ilharreborde B, Fournier J, et al. Adolescent idiopathic scoliosis correction achieved by posteromedial translation using polyester bands: a comparative study of subtransverse process versus sublaminar fixation. Orthop Traumatol Surg Res 2014;100:791-5.

12. Ilharreborde B, Sebag G, Skalli W, et al. Adolescent idiopathic scoliosis treated with posteromedial translation: radiologic evaluation with a 3D low-dose system. Eur Spine J 2013;22:2382-91.

13. Hu P, Yu M, Liu X, et al. Analysis of the relationship between coronal and sagittal deformities in adolescent idiopathic scoliosis. Eur Spine J 2016;25:409-16.

14. Mac-Thiong JM, Labelle H, Charlebois M, et al. Sagittal plane analysis of the spine and pelvis in adolescent idiopathic scoliosis according to the coronal curve type. Spine 2003;28:1404-9.

15. Upasani VV, Tis J, Bastrom T, et al. Analysis of sagittal alignment in thoracic and thoracolumbar curves in adolescent idiopathic scoliosis: how do these two curve types differ? Spine 2007;32:1355-59.

16. Cheng I, Kim Y, Gupta MC, et al. Apical sub-laminar wires versus pedicle screws - Which provides better results for surgical correction of adolescent idiopathic scoliosis? Spine 2005;30:2104-12.

17. Ogawa H, Hori H, Oshita H, et al. Sublaminar wiring stabilization to prevent adjacent segment degeneration after lumbar spinal fusion. Arch Orthop Trauma Surg 2009;129:873-8.

18. Sales de Gauzy J, Jouve JL, Ilharreborde B, et al. Use of the Universal Clamp in adolescent idiopathic scoliosis. Eur Spine J 2014;23:S446-51.

19. La Rosa G, Giglio G, Oggiano L. The Universal Clamp hybrid system: a safe technique to correct deformity and restore kyphosis in adolescent idiopathic scoliosis. Eur Spine J 2013;22:S823-8.

20. Palmisani M, Dema E, Cervellati S, et al. Hybrid constructs pedicle screw with apical sublaminar bands versus pedicle screws only for surgical correction of adolescent idiopathic scoliosis. Eur Spine J 2018;27:150-6.

21. Fletcher ND, Hopkins J, McClung A, et al. Residual thoracic hypokyphosis after posterior spinal fusion and instrumentation in adolescent idiopathic scoliosis: risk factors and clinical ramifications. Spine 2012;37:200-6.

22. Angelliaume A, Ferrero E, Mazda K, et al. Titanium vs cobalt chromium: what is the best rod material to enhance adolescent idiopathic scoliosis correction with sublaminar 
bands? Eur Spine J 2017;26:1732-38.

23. Battista C, Wild C, Kreul S, Albert M. Prevention of Proximal Junctional Kyphosis \& Failure Using Sublaminar Bands in a Hybrid Construct in Pediatric Kyphosis Deformity. Int J Spine Surg 2018;12:644-9.

24. Protopsaltis TS, Scheer JK, Terran JS, et al. How the neck affects the back: changes in regional cervical sagittal alignment correlate to HRQOL improvement in adult thoracolumbar deformity patients at 2-year follow-up. J Neurosurg Spine 2015;23:153-8.

25. Blondel B, Lafage V, Schwab F, et al. Reciprocal sagittal alignment changes after posterior fusion in the setting of adolescent idiopathic scoliosis. Eur Spine J 2012;21:1964-71.

26. Corradin M, Canavese F, Dimeglio A, et al. Cervical sagittal alignment variations in adolescent idiopathic scoliosis patients treated with thoraco-lumbo-sacral orthosis. Eur Spine J 2017;26:1217-24.

27. Canavese F, Turcot K, DeRosa V, et al. Cervical spine sagittal alignement variations following posterior fusion and instrumentation for adolescent idiopathic scoliosis. Eur Spine J 2011;20:1141-8.

28. Charles YP, Sfeir G, Matter-Parrat V, et al. Cervical sagittal alignment in idiopathic scoliosis treated by instrumentation and in situ bending. Spine (Phila $\mathrm{Pa} 1976$ ) 2015;40:E419-27.

29. Albert MC, LaFleur BC. Hybrid fixation with sublaminar polyester bands in the treatment of neuromuscular scoliosis: a comparative analysis. J Pediatr Orthop 2015;35:172-7.

30. Desai SK, Sayama C, Vener D, et al. The feasibility and safety of using sublaminar polyester bands in hybrid spinal constructs in children and transitional adults for neuromuscular scoliosis. J Neurosurg Pediatr 2015;15:328-37.

Cite this article as: Canavese F, Samba A. Sublaminar polyester bands for the correction of idiopathic and neuromuscular scoliosis. Ann Transl Med 2020;8(2):32. doi: 10.21037/atm.2019.08.109
31. La Rosa G, Giglio G, Oggiano L. Surgical treatment of neurological scoliosis using hybrid construct (lumbar transpedicular screws plus thoracic sublaminar acrylic loops). Eur Spine J 2011;20 Suppl 1:S90-4.

32. La Rosa G, Giglio G, Oggiano L. Sagittal profile control in patients affected by neurological scoliosis using Universal Clamps: a 4-year follow-up study. Eur Spine J 2012;21 Suppl 1:S32-6.

33. Canavese F, Rousset M, Le Gledic B, et al. Surgical advances in the treatment of neuromuscular scoliosis. World J Orthop 2014;5:124-33.

34. Tsirikos AI, Chang WN, Dabney KW, et al. Comparison of one-stage versus two-stage anteroposterior spinal fusion in pediatric patients with cerebral palsy and neuromuscular scoliosis. Spine (Phila Pa 1976) 2003;28:1300-5.

35. Master DL, Son-Hing JP, Poe-Kochert C, et al. Risk factors for major complications after surgery for neuromuscular scoliosis. Spine (Phila Pa 1976) 2011;36:564-71.

36. Lonstein JE, Koop SE, Novachek TF, et al. Results and Complications After Spinal Fusion for Neuromuscular Scoliosis in Cerebral Palsy and Static Encephalopathy Using Luque Galveston Instrumentation: Experience in 93 Patients. Spine 2012;37:583-91.

37. Issa SP, Angelliaume A, Vidal C, et al. Do Sublaminar Polyester Bands Affect the Outcomes of Postoperative Infections After Adolescent Idiopathic Scoliosis Surgery? J Pediatr Orthop 2017;37:e524-9.

38. Canavese F, Gupta S, Krajbich JI, et al. Vacuum-assisted closure for deep infection after spinal instrumentation for scoliosis. J Bone Joint Surg Br 2008;90:377-81.

39. Canavese F, Marengo L, Corradin M, et al. Deep postoperative spine infection treated by negative pressure therapy in patients with progressive spinal deformities. Arch Orthop Trauma Surg 2018;138:463-9. 